Schwerpunkt

Herzschr Elektrophys 2020 • 31:265-272 https://doi.org/10.1007/s00399-020-00707-6 Eingegangen: 28. April 2020

Angenommen: 3. Juli 2020

Online publiziert: 5 . August 2020

(c) Springer Medizin Verlag $\mathrm{GmbH}$, ein Teil von Springer Nature 2020

Im Rahmen der Digitalisierung besteht die Möglichkeit, Herzrhythmusstörungen in einem relevanten Umfang über sog. Implantables und Wearables zu detektieren. Diese Geräte produzieren eine erhebliche Datenmenge und geben einen Einblick in das, was in unserem Körper vor sich geht. Es ist nicht überraschend, dass diese Geräte zunehmend an Popularität gewinnen, da viele Menschen nun die direkte Möglichkeit haben, individuelle Veränderungen, Fortschritte, aber auch Rückschritte kontinuierlich und quantitativ zu erfassen und darauf zu reagieren.

Tragbare Geräte wie Fitness-Tracker und Smartwatches, die in der Vergangenheit während des körperlichen Trainings verwendet wurden, spielen jetzt eine Rolle bei der Kontrolle von Risikofaktoren und weisen auf Abweichungen von definierten Parametern, wie z. B. Herzrhythmusstörungen, hin. Darüber hinaus ermöglichen diese Devices über Fern- und Telemonitoring in der heutigen Zeit, die aktuell von der COVID-19-Pandemie geprägt ist, eine sichere und unproblematische Anbindung von Arrhythmiepatienten.

In den maßgebenden Studien EMBRACE (Event Monitor Belt for Recording Atrial Fibrillation after a Cerebral Ischemic Event; NCT00846924; [1]) und CRYSTAL-AF (Study of Continuous Cardiac Monitoring to Assess Atrial Fibrillation After Cryptogenic Stroke; NCT00924638; [2]) konnte ein signifikanter Vorteil des kontinuierlichen Rhythmusmonitorings mittels Brustgurt bzw. implantierbarem Ereignisrekorder

Christian Sohns · Moneeb Khalaph · Leonard Bergau · Philipp Sommer

Klinik für Elektrophysiologie/Rhythmologie, Herz- und Diabeteszentrum Nordrhein-Westfalen, Ruhr Universität Bochum, Bad Oeynhausen, Deutschland

\title{
Smart and simple
}

\section{Stellenwert von Implantables und Wearables im rhythmologischen Alltag}

bei Patienten mit kryptogenem Schlagfanfall in Bezug auf die Detektion von Vorhofflimmern im Vergleich zu konventionell geführten Patienten gezeigt werden. In der EMBRACE-Studie wurden 572 Patienten (Alter $>55$ Jahre) mit kryptogenem Schlaganfall entweder einer 24-Stunden-EKG-Untersuchung (Kontrollgruppe) oder einer 30-tägigen nichtinvasiven Rhythmusüberwachung mithilfe eines Brustgurts unterzogen. In der CRYSTAL-AF-Studie wurden 441 Patienten in eine Gruppe mit implantierbarem Ereignisrekorder (Medtronic Reveal XT ${ }^{\mathrm{m}}$ [Medtronic Inc., Minneapolis, MN, USA]) oder eine LangzeitEKG-Gruppe randomisiert. Über einen Beobachtungszeitraum von 30 Tagen wurden in der EMBRACE-Studie signifikant mehr Vorhofflimmerepisoden detektiert als in der konventionellen Gruppe $(16,1 \%$ vs $3,2 \%, p<0,001)$. In der CRYSTAL-AF-Studie zeigte sich über dem gesamten Beobachtungszeitraum eine signifikant bessere Detektionsrate des implantierbaren Ereignisrekorders im Vergleich zum konventionellen Langzeit-EKG $(8,9 \%$ vs. $1,4 \%$ nach 6 Monaten; $12,4 \%$ vs. $2,0 \%$ nach 12 Monaten und $30 \%$ vs. $3,0 \%$ nach 3 Jahren).

Es ist davon auszugehen, dass Implantables und Wearables in der modernen Medizin zunehmend zur Anwendung kommen werden, um ein kontinuierliches Monitoring von relevanten Messgrößen und Parametern zu gewährleisten. Darüber hinaus kann der Einsatz von diesen Devices aber auch dazu beitragen, das subjektive Bewusstsein und individuelle Engagement der Patienten hinsichtlich ihrer Compliance zu erhöhen. Aber auch wir behandelnden Ärzte oder medizinisches Fachpersonal haben einen relevanten Nutzen hinsichtlich der Patientenversorgung durch die Verwendung dieser Geräte, da wir in Echtzeit Informationen über unsere Patienten bekommen und somit evtl. sogar schon vor dem Auftreten von klinischen Symptomen angemessen auf eine Situation reagieren können.

Anlässlich der Consumer Electronics Show (CES) in Las Vegas hat die elektrophysiologische US-Fachgesellschaft Heart Rhythm Society (HRS) mit dem CES-Veranstalter, der Consumer Trade Association (CTA), ein gemeinsames Positionspapier $\mathrm{zu}$ Wearables vorgestellt [3]. Hintergrund ist die steigende Nutzerakzeptanz der o.g. MonitoringDevices. Mit dem Positionspapier macht die HRS deutlich, dass sie den Trend zu Wearables prinzipiell unterstützt und sehr viel Positives hinsichtlich einer früheren Diagnose und einem besseren Management von Erkrankungen gesehen wird [3]. Dieser Artikel zeigt anhand einiger ausgewählter Beispiele die Anwendung und Funktionalität von Monitoring-Devices und soll auf diesem Wege den potenziellen Stellenwert von Implantables und Wearables in unserem klinischen elektrophysiologischen Alltag diskutieren.

\section{Implantables}

Viele unterschiedliche Parameter können durch implantierte Devices erhoben werden. In diesem Abschnitt soll ein besonderes Augenmerk auf implantierbare Herzmonitore gelegt werden. Im Folgenden werden aktuelle Modelle inklusive 


\section{Schwerpunkt}
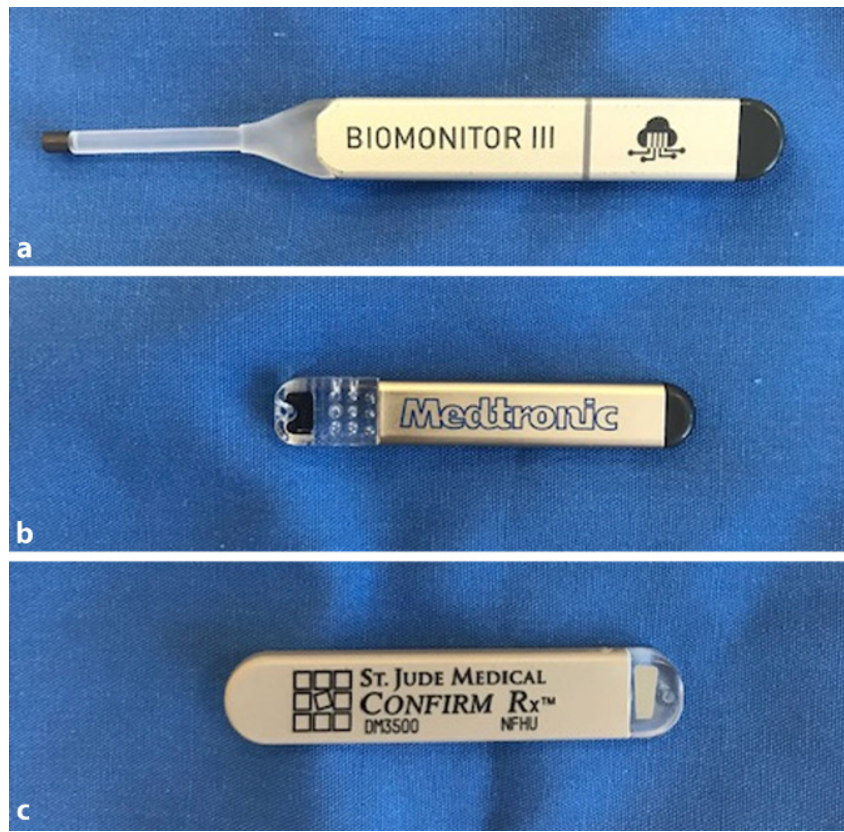

Abb. $1<$ Beispielhafte Darstellung von Demoversionen: a BioMonitor III ${ }^{\mathrm{TM}}$, b Reveal $\mathrm{LINQ}^{\mathrm{TM}}$ und c Confirm $\mathrm{Rx}^{\mathrm{TM}}$

ihrer Anwendung und Anbindung repräsentativ vorgestellt.

\section{Medtronic Reveal LINQ ${ }^{\mathrm{TM}}$}

Der Reveal LINQ ${ }^{\text {rm }}$ (Medtronic Inc., Minnesota, MN, USA) stellt die neueste Generation von Ereignisrekordern der Firma Medtronic dar. Er dient der Detektion von tachykarden und bradykarden Arrhythmien. Ein besonderer Schwerpunkt liegt auf der Dokumentation von Vorhofflimmern, aber es können ebenso ventrikuläre Arrhythmien, bradykarde Phasen und Überleitungsstörungen erfasst werden. Der Reveal LINQ ermöglicht die kontinuierliche Speicherung von Elektrogrammen direkt während des Auftretens von Rhythmusereignissen, die entweder automatisch erkannt werden oder aktiv vom Patienten (durch die Symptome getriggert) dokumentiert werden können. Die automatische Aktivierung im Sinne einer individualisierten Monitorzone ist vorteilhaft, um asymptomatische oder oligosymptomatische Episoden zur erfassen oder um Arrhythmien bei Patienten zu sichern, die das Device nicht selbst aktivieren können. Der Reveal LINQ $^{\mathrm{Tm}}$ ist deutlich kleiner als seine Vorgänger und hat ein Gewicht von $15 \mathrm{~g}$ (• Abb. 1). Während eines unkomplizierten Eingriffs kann das Device unter örtlicher Betäubung in der Regel parasternal rechts subkutan implantiert werden. Die durchschnittliche Laufzeit beträgt 3 Jahre, das Device ist MRT-tauglich bis $3 \mathrm{~T}$.

In der Vergangenheit sind durch Vorgängermodelle vereinzelt falsch-positive Episoden von Herzrhythmusstörungen aufgezeichnet worden [4]. Dies ist insbesondere bei der Detektion von Vorhofflimmern der Fall gewesen. Durch eine submuskuläre Implantation konnte die Signalqualität und Detektion von appropriaten Episoden verbessert werden [5]. Die technische Weiterentwicklung hat die Detektion von Arrhythmien durch den Reveal LINQ ${ }^{\mathrm{Tw}}$ an den gängigen Implantationsstellen deutlich verbessert [6]. Neben den technisch-sensorischen Erneuerungen lässt sich das neue Device auch problemlos an telemedizinische Zentren anbinden und ermöglicht somit eine schnelle Reaktion auf die klinisch dokumentierte Situation. Ferner kann durch die telemedizinische Anbindung auch die Anzahl an unnötigen ambulanten Vorstellungen reduziert werden $[7,8]$.

\section{Abbott Confirm Rx ${ }^{\mathrm{TM}}$}

Der Confirm $\mathrm{Rx}^{\mathrm{mm}}$ (Abbott, Chicago, IL, USA; Abb. 1) ist ebenfalls ein sehr einfach zu implantierender Ereignisrekorder der Firma Abbott (ehemals St. Jude Medical). Dieses Rhythmusdevice ermöglicht die automatische und kontinuierliche Detektion von Herzrhythmusstörungen. Das Vorgängermodell, der SJM Confirm AF ${ }^{\text {rix }}$ (Abbott, Chicago, IL, USA) hat sich in der Vergangenheit sowohl in der Synkopen- als auch in der Vorhofflimmerdiagnostik bei Patienten mit kryptogenem Schlaganfall bewährt $[9,10]$. In diesem Zusammenhang ist die Detect-AF-Studie (Enhanced Diagnostics for Early Detection of Atrial Fibrillation; NCT02270112) hervorzuheben, welche die Zuverlässigkeit des Devices hinsichtlich der Detektion und des kontinuierlichen Monitorings bei Patienten mit bekanntem oder vermutetem Vorhofflimmern nachgewiesen hat [10]. Aktuell untersucht das Confirm $\mathrm{Rx}$ Insertable Cardiac Monitor SMART Register (NCT03505801) Implantations- und Follow-up-Daten zum Device. Die Episoden können ebenfalls automatisiert als Rhythmusmonitor erfasst werden. Eine aktive Speicherung durch den Patienten ist bei Symptomen ebenfalls möglich. Der Confirm $\mathrm{Rx}^{\mathrm{Tm}}$ ist zur kontinuierlichen Überwachung und diagnostischen Beurteilung von Patienten mit bislang ungeklärten Symptomen vorgesehen (z. B. Schwindel, Palpitationen, Brustschmerzen, Synkopen) sowie zum Monitoring von Patienten mit einem Risiko oder einer Anamnese von Herzrhythmusstörungen. Er ist auch für Patienten vorgesehen, bei denen bereits Vorhofflimmern diagnostiziert wurde oder bei denen ein erhöhtes Risiko für Vorhofflimmern besteht. Der Confirm $\mathrm{Rx}^{\mathrm{Tm}}$ hat eine durchschnittliche Batterielaufzeit von ca. 2 Jahren und ist MRTtauglich. Als weiteres Feature ist er Bluetooth-kompatibel. Interessant ist auch die Möglichkeit der Fernabfrage über die Smartphone-App myMerlin ${ }^{\mathrm{Tm}}$. Der zuständige Arzt erhält hierüber die benötigten Elektrogramme und Rhythmusinformationen über die telemedizinische Anbindung. Neben der kontinuierlichen Rhythmusüberwachung ermöglicht die myMerlin $^{\text {nx }}$ Smartphone-App auch, aktiv symptomatische Episoden aufzuzeichnen und an das angebundene Zentrum zu übermitteln. 


\section{Biotronik BioMonitor III ${ }^{\mathrm{TM}}$}

Der BioMonitor III ${ }^{\text {mix }}$ (Biotronik, Berlin, Deutschland, Abb. 1) ist der neueste Rhythmusmonitor der Firma Biotronik. Wie die beiden o.g. Devices ist eine einfache und sichere subkutane Implantation unter Lokalanästhesie generell möglich. Der BioMonitor ist in der Lage, Elektrogramme und physiologische Parameter kontinuierlich aufzuzeichnen. Das Vorgängermodell, das 3 sensorische Vektoren zur Signalerkennung verwendet, ist überwiegend in Studien zur Detektion von Vorhofflimmern evaluiert worden [11-13]. Arrhythmien werden automatisch erfasst und können auch aktiv durch den Patienten gespeichert werden. Im Vergleich zu seinem Vorgängermodell ist das aktuelle Device um ca. $60 \%$ kompakter und kleiner. Der BioMonitor III ${ }^{\mathrm{Tm}}$ ermöglicht ebenfalls eine direkte Fernüberwachung durch Anbindung an die Home Monitoring ${ }^{\circledR}$-Technologie. Die BIO CONCEPT.BIOMONITOR IIIStudie (NCT03850327) rekrutiert aktuell Patienten, um die Sicherheit (inklusive der Implantation) und Effektivität des BioMonitor III $^{\mathrm{Tw}} \mathrm{zu}$ evaluieren. Der BioMonitor III ${ }^{\mathrm{Tm}}$ ist MRT-fähig und hat eine durchschnittliche Laufzeit von 4 Jahren.

In der Herstellerinformation wird das neue BIO-Vector Design hinsichtlich der Qualität der Signalamplituden und einer vergrößerten Sensing-Oberfläche hervorgehoben [14]. Der BioMonitor III ${ }^{\mathrm{sm}}$ könne somit sehr zuverlässig Vorhofund Ventrikelsignalamplituden erfassen. Dies soll viele falsch-positive Episoden vermindern. Auch hier findet die Übermittlung anhand einer Smartphone-App (Biotronik - Patient App) statt. Der Patient kann auch zusätzliche Angaben $\mathrm{zu}$ seinem Gesundheitszustand über die App erfassen. Diese beinhalten auch ein Symptomtagebuch, welches umfassende Informationen für Patienten und Ärzte bereitstellt. Die App ermöglicht auch eine direkte Kommunikation mit dem Ansprechpartner am telemedizinischen Zentrum.

\section{Wearables}

Aktuelle Daten zeigen, dass kontinuierliches Monitoring eine relevante Verbes-

Herzschr Elektrophys 2020 · 31:265-272 https://doi.org/10.1007/s00399-020-00707-6

(c) Springer Medizin Verlag GmbH, ein Teil von Springer Nature 2020

\section{Sohns $\cdot$ M. Khalaph $\cdot$ L. Bergau $\cdot$ P. Sommer}

\section{Smart and simple. Stellenwert von Implantables und Wearables im rhythmologischen Alltag}

\section{Zusammenfassung}

In den letzten Jahren hat die Verwendung von Implantables und Wearables zum Monitoring von Herzfrequenz und -rhythmus stetig zugenommen. Inzwischen stehen smarte und einfache Lösungen zur Verfügung, um den Herzrhythmus kontinuierlich zu überwachen und die dokumentierten Daten weiterzuleiten. Aus diesem Grund können Implantables und Wearables auch einfach und direkt an telemedizinische Zentren angebunden werden. Als große Herausforderung für den verstärkten Einsatz von Monitoring-Devices gilt weiterhin eine relativ ungefilterte Datenflut. Dieser Übersichtsartikel zeigt anhand einiger ausgewählter Beispiele die Anwendung und Funktionalität von Monitoring-Devices und soll auf diesem Wege den potenziellen Stellenwert von Implantables und Wearables in unserem klinischen elektrophysiologischen Alltag darstellen und differenziert diskutieren.

Schlüsselwörter

Herzrhythmusstörungen - Vorhofflimmern . Monitoring $\cdot$ Signalqualität $\cdot$ Reaktionszeit

\section{Smart and simple. Current role of implantables and wearables in daily practice}

Abstract

Recent developments indeed point towards implantable and wearables, especially smartwatches, as being quite effective and a novel method for screening of arrhythmias in the general population or rhythm monitoring in our patients. The new generation of smart rhythm monitoring devices allows for continuous monitoring and simple real time access to the majority of the data. Therefore, they are well suited to be part of the growing number of so-called remote medicine concepts. Although the technology is not only restricted to smartwatches, single lead ECGs that are portable and phone applications that detect heart rate can serve as event recorders during an episode of symptoms. Furthermore, implanted rhythm monitoring may also communicate with mobile applications to allow more rapid and accurate arrhythmia diagnosis and therapy. The authors aimed to demonstrate and discuss new technical developments focusing on the latest generation of implantables and wearables and their benefit in terms of smart and simple cardiac rhythm monitoring.

\section{Keywords}

Cardiac arrhythmias - Atrial fibrillation . Monitoring · Signal quality · Reaction time serung der Detektion von Herzrhythmusstörungen ermöglicht [15-17]. Viele Wearables sind ideal für ein kontinuierliches Monitoring geeignet, da sie über einen langen Zeitraum direkt am Körper getragen werden. Zusätzlich werden die gemessenen Parameter gespeichert und sind permanent abrufbar. Einige Devices können somit auch telemedizinisch angebunden werden, sofern sie als $\mathrm{Me}$ dizinprodukte zugelassen sind oder im Rahmen von Studien evaluiert werden. Im Folgenden soll der aktuelle Stellenwert von Wearables exemplarisch anhand der Apple Heart Study gestellt werden.

\section{Smartwatches - Apple Watch und} Apple Heart Study

Derzeit wird viel darüber diskutiert, ob und wie Apps und etwa am Handgelenk getragene Wearables wie Smartwatches als digitale Helfer in der Medizin genutzt werden können. Wearables nutzen zunehmend Sensoren, die ohnehin täglich am Körper getragen werden. Ein allgegenwärtiges Beispiel ist die Apple Watch, stellvertretend genannt für andere Smartwatch-Devices. Die Applikationen, welche die Apple Watch zur Beurteilung des Pulses und zur Darstellung des EKGs ver- 


\section{Schwerpunkt}
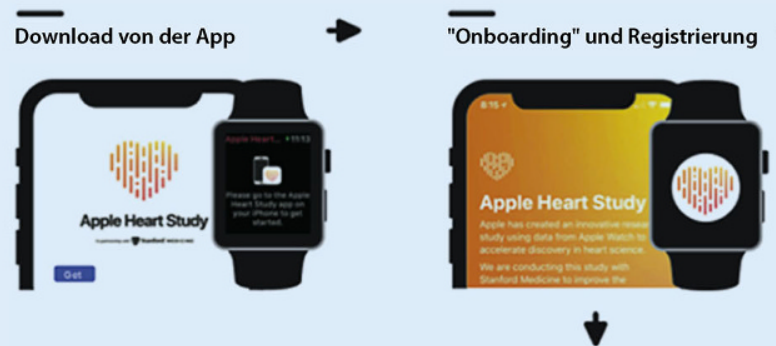

Normale Tachogramme

Keine Benachrichtigung ausgelöst Ende des Studienfragebogens

Unregelmäßige Tachogramme

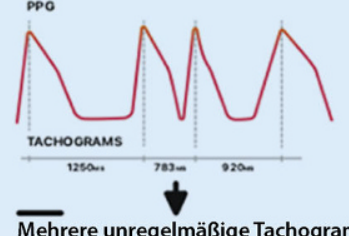

Mehrere unregelmäßige Tachogramme lösen eine Benachrichtigung wegen eines unregelmäßigen Herzrhythmus aus (Irregular Heart Rhythm Notification)

- mocurarioman

- posseucus

PPO
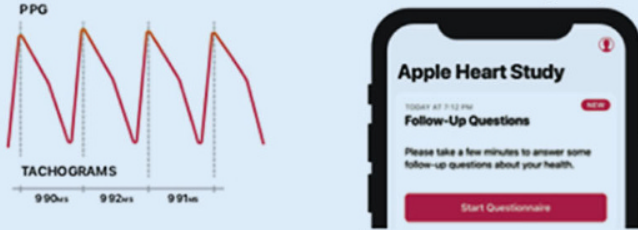
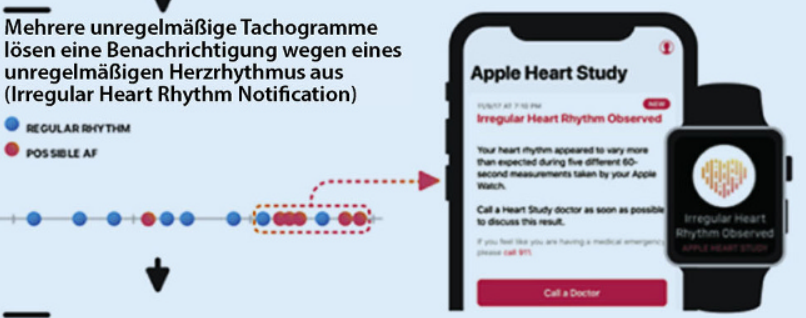

Erster Video-Besuch mit dem Studienarzt
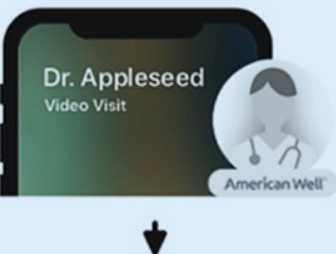

Geeignet für ePatch

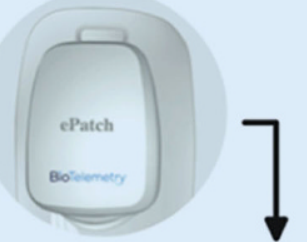

Kein AF/andere Arrhythmien auf dem ePatch-Bericht

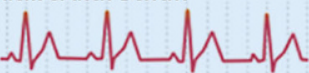

\section{$\downarrow$}

Empfehlung an den Teilnehmer, mit seiner gewohnten Versorgung fortzufahren
Zweiter Video-Besuch mit dem Studienarzt
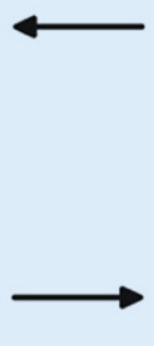
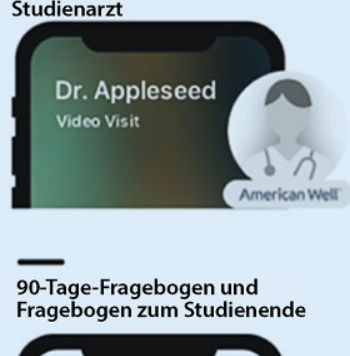

Apple Heart Study

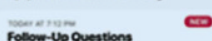

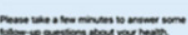
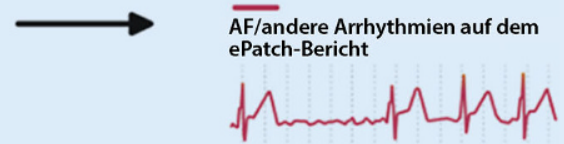

文

Empfehlung an den Patienten, seinen Gesundheitsversorger zu kontaktieren

Abb. $2 \Delta$ Apple Heart Studie - repräsentative Abbildung zum technischen Ablauf der Informationsverarbeitung. AF Vorhofflimmern. (Nach [26], mit freundlicher Genehmigung von Elsevier) 
Tab. 1 Sensitivität, Spezifität, positiv-prädiktiver Wert (PPW), und negativ-prädiktiver Wert (NPW) von repräsentativen Devices hinsichtlich der Detektion von Vorhofflimmern. (Mod. nach [24])

\begin{tabular}{l} 
Device \\
\hline Cardio Rhythm App \\
Apple Watch (von der \\
FDA zugelassen) \\
Kardia Band (von der \\
FDA zugelassen) \\
Alive Cor single lead EKG \\
(von der FDA \\
zugelassen)
\end{tabular}

Studien

Chan et al., 2016 [25]

Turakhia et al., 2019 [26]

Bumgarner et al., 2018 [27]

Chan et al., 2016 [25] Desteghe et al., 2017 [28]

Koshy et al., 2018

[29]

My Diagnostik Desteghe et al., 2017 [28]

\section{Sensitivität/Spezifizität/PPW/NPW}

Sensitivität: 92,9\%; Spezifizität: 97,7\%; PPW: 53,1\%; NPW: 99,8\%

PPW des Tachograms: $71 \%$; PPW der Benachrichtigung: $84 \%$

Sensitivität: $93 \%$; Spezifität: $84 \%$

Sensitiviät: 71,4\%, Spezifität: 99,4\%; PPW: 76,9\%; NPW: 99,2\%

Sensitivität: 54,5\%; Spezifität: 97,5\% (bei kardiologischen Patienten)

Sensitivität: 78,9\%; Spezifität: 97,9\% (bei geriatrischen Patienten)

Sensitivität: $77 \%$; Spezifität: 76\%

Sensitivität: 81,8\%; Spezifität: 94,2\% (bei kardiologischen Patienten)

Sensitivität: 89,5\%; Spezifität: 95,7\% (bei geriatrischen Patienten)

Tab. 3 Vor- und Nachteile von smartem Monitoring durch Implantables und Wearables

Vorteile Sofortiger Zugriff aufEchtzeit-Gesundheitsdaten möglich

Motivation und Ermutigung zum Monitoring durch smarte Applikationen

Gestärktes Verantwortungsbewusstsein aufgrund der kontinuierlichen Kontrolle

Möglichkeit zur Früherkennung von akuten und chronischen Erkrankungen, bevor Symptome auftreten

Vereinfachter Arzt-Patienten-Kontakt

Verbesserte klinische Entscheidungsfindung

Nach- Unterschiedliche Datengenauigkeit und Möglichkeit für falsch-positive Messwerte

teile Fehlender Nachweis eines gesundheitlichen Benefits

Eingeschränkte Erreichbarkeit aufgrund von zusätzlichen Kosten oder fehlender Netzkapazitäten

Datenflut für den Anwender und das telemedizinische Zentrum

Noch kein Einbezug der Gesundheitsakte möglich

Offene Fragen hinsichtlich der Datensicherheit und des Datenschutzes

wendet, wurden 2018 von der FDA als Klasse-II-Produkt eingestuft.

Im letzten Jahr wurde die vieldiskutierte Apple Heart Study veröffentlicht [18]. In dieser Studie, an der 419.093 Personen beteiligt waren, sollten neue Möglichkeiten zum Screening bzw. zur Detektion von zuvor nicht bekanntem Vorhofflimmern evaluiert werden. Zugelassen zur Studie wurden Personen, die mindestens 22 Jahre alt und US-Bürger waren. Ferner mussten alle Teilnehmer Träger einer Apple Watch sein sowie ein iPhone mit installierter Apple Heart Study App besitzen. Das Ziel der Studie war es, zu erheben, wie viele Personen, die wegen detektierter PulsunregelmäBigkeiten eine Benachrichtigung via App erhielten, während eines nachfolgenden ambulanten EKG-Monitorings tatsächlich Vorhofflimmern hatten. Zudem sollte der positiv-prädiktive Wert der Mitteilung von Pulsunregelmäßigkeiten per Abgleich mit den Aufzeichnungen eines gleichzeitigen EKG-Monitorings bestimmt werden. Darüber hinaus wollte man beobachten, was Teilnehmer mit erhaltener Benachrichtigung etwa bezüglich der weiteren medizinischen Abklärung in den folgenden 3 Monaten von sich aus unternommen haben und welche Ressourcen im Gesund-
Tab. 2 Überblick hinsichtlich der un-

terschiedlichen Arten der Datenerfas-

sung durch Implantables und Wearables.

(Mod. nach [3])

\section{Arten der Datenerfassung}

1. Randomisierte oder regelmäßige Datenerfassung

Über Sensoren, die wir am Körper tragen, werden schon seit Jahren randomisiert oder regelmäßig Daten erfasst

Die Anwendung von digitalen Technologien ermöglicht eine simple und effiziente Datenspeicherung, -überwachung und -nachverfolgung

Wearables ermöglichen häufigere oder auch kontinuierliche Messungen, um Änderungen im Verlauf zu darzustellen

2. Intermittierende oder symptomgesteuerte Datenerfassung (für Patienten mit Symptomen)

Dieses Messverfahren setzt intermittierende Symptome voraus, die zu unterschiedlichen Zeitpunkten erfasst werden

Es besteht meist auch die Möglichkeit, dass Benutzer aktiv (symptombezogen) die Datenerfassung aktivieren und deaktivieren können

Diese Form der Datenerhebung kann z. B. die Diagnosestellung unterstützen, ein lückenloses Follow-up ermöglichen oder das Ansprechen einer Behandlung widerspiegeln

\section{Kontinuierliche Datenerhebung}

Eine kontinuierliche Datenerhebung läuft permanent und uneingeschränkt mit, um dauerhaft Informationen aufzuzeichnen. Somit können z. B. tägliche Aktivitäten, Schlafmuster oder Vitalparameter dokumentiert werden

Eine Aktivierung durch den Benutzer ist nicht notwendig, die Dokumentation erfolgt passiv durch das Device

Insbesondere bei Patienten mit Herzrhythmusstörungen hat die kontinuierliche Datenerhebung einen hohen Stellenwert und möglicherweise einen prognostischen Effekt

heitswesen dafür genutzt wurden. Im Fall der mehrfachen Detektion von unregelmäßigem Puls durch die Apple Heart Study App (5 von 6 Tachogrammen mussten unregelmäßig sein), erhielt der Teilnehmer vom telemedizinischen Servicezentrum der Studie eine entsprechende Benachrichtigung. Man hatte danach die Möglichkeit, per App Kontakt mit einem „Telehealth Provider" der Studie aufzunehmen und ein SensorDevice zur Langzeit-EKG-Kontrolle zu 


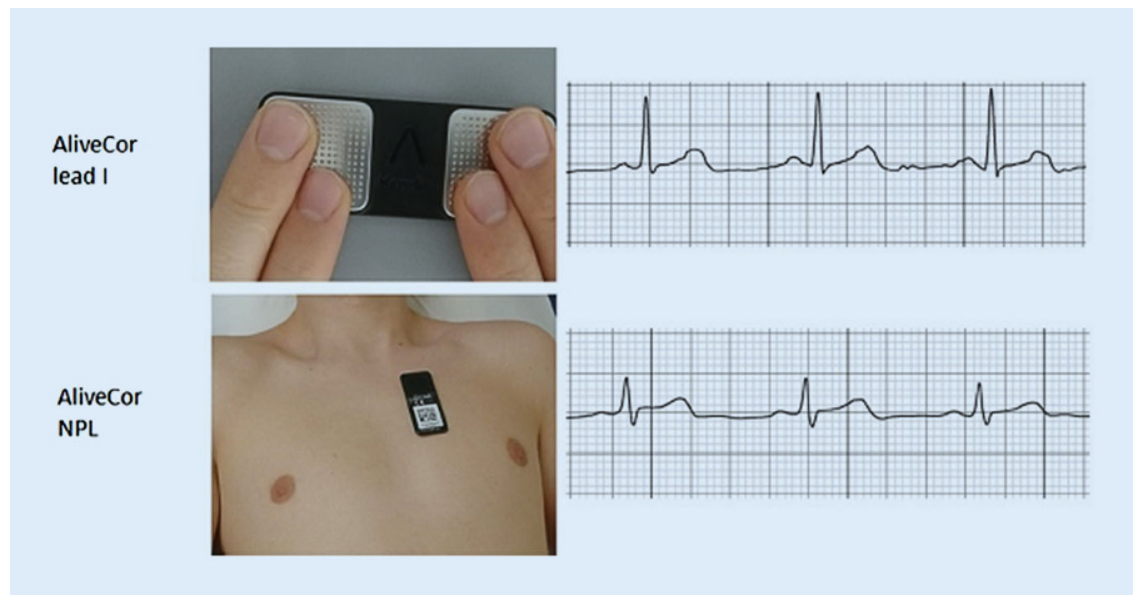

Abb. $3 \Delta$ Anwendung des Kardia EKG Monitors und repräsentative Beispiele für EKG-Ableitungen von Ableitung I (oben) und einer parasternalen Ableitung (unten). (Nach [19], mit freundlicher Genehmigung von Elsevier)

erhalten (•Abb. 2). Von allen kontinuierlich per Apple Watch überwachten Patienten erhielten etwa 0,52\% die Benachrichtigung eines unregelmäßigen Herzschlags. Dies ist möglicherweise darauf zurückzuführen, dass das durchschnittliche Alter der Studienteilnehmer bei 41 Jahren lag und somit die Inzidenz von anhaltenden Tachyarrhythmien bzw. von Vorhofflimmern in diesem Kollektiv ohnehin relativ gering ist. Mit 3,1\% war der Anteil in der Subgruppe der über 65-Jährigen $(n=24.626)$ noch relativ am höchsten, während in der weitaus größeren Gruppe der unter 40-Jährigen sehr selten $(0,16 \%)$ eine Warnmeldung verschickte wurde. Von den benachrichtigten Teilnehmern hatten 658 einen externen EKG-Monitor zugeschickt bekommen, und 450 von diesen Personen haben das Device dann auch zur Auswertung zurückgesendet. Hierbei ließ sich bei 153 der 450 Teilnehmer (34\%) im EKG tatsächlich Vorhofflimmern dokumentieren. Von den 450 Probanden, bei denen der Herzrhythmus gleichzeitig von der Apple Watch und dem EKGMonitor aufgezeichnet wurde, hatten 86 in dieser Zeit eine Meldung über unregelmäßigen Herzschlag erhalten. Bei 72 von 86 Personen war zeitgleich Vorhofflimmern im EKG dokumentiert worden. Daraus resultierte ein positivprädiktiver Wert von 0,84 für die Benachrichtigung durch die SmartphoneApp. Die Autoren der Studie fassten zusammen, dass die Apple Watch im
Sinne einer Wearable-Technologie Arrhythmien sicher identifizieren kann und somit insbesondere bei Vorhofflimmern ein wichtiges Tool hinsichtlich der Detektion von Arrhythmie-Episoden sein kann. Weitere Informationen in diesem Zusammenhang bietet $\bullet$ Tab. 1 .

\section{Kardia EKG Monitor - AliveCor}

Der Kardia EKG Monitor von der Firma AliveCor (Mountain View, CA, USA) ist ein tragbares Gerät zur Beurteilung des Herzrhythmus durch ein 1- bzw. 6-KanalEKG. Anders als die o. g. Devices wird es nicht implantiert und auch nicht kontinuierlich auf der Haut getragen. Wie in - Abb. 3 gezeigt, kann mit dem Device über eine Smartphone-App eine symptomatische Episode von Herzrhythmusstörungen aktiv aufgezeichnet werden. Das Device hat ungefähr die Größe eines Feuerzeugs und kann somit leicht in der Tasche getragen werden.

Das Device ist ebenfalls von der FDA geprüft und als Klasse-II-Produkt eingestuft worden. Im Rahmen der aktuell bestehenden COVID-19-Krise wurde zudem eine QTc-Messmethode des 6-Kanal-EKGs geprüft und ebenfalls zugelassen. Eine aktuelle prospektive Studie zur Verwendung des Kardia EKG Monitors bei 99 Patienten hat gezeigt, dass symptomatische Episoden von Vorhofflimmern mit hoher diagnostischer Qualität erfasst werden können [19]. Daher sehen die Autoren ein geeig- netes Einsatzgebiet des 1-Kanal EKGMonitors beim Screening von Patienten mit Vorhofflimmern. Bei Patienten mit atrialen Tachykardien oder Trägern von Herzschrittmachern fand man noch technische Limitationen [19]. Die aktuell noch laufende MOBILE-AF-Studie evaluiert den diagnostischen Stellenwert des Kardia EKG Monitor gegenüber einem implantierten Ereignisrekorder hinsichtlich der Detektion von Vorhofflimmern bei Patienten mit kryptogenem Schlaganfall [20]. Die Ergebnisse dieser Studie könnten einen Hinweis darauf geben, ob zukünftig mobile Applikationen bei selektierten Patienten die Implantation eines kontinuierlichen Rhythmusmonitors ersetzen können. In diesem Zusammenhang zeigt $\bullet$ Tab. 1 eine Übersicht hinsichtlich Sensitivität, Spezifität, negativ- und positiv-prädiktivem Wert für die Detektion von Vorhofflimmern durch repräsentativ ausgewählte Wearables.

\section{Diskussion und Ausblick}

In den letzten Jahren hat die Verwendung von Implantables und Wearables zum Monitoring von Herzfrequenz und -rhythmus durch unsere Patienten stetig zugenommen. Unabhängig davon zeigt uns nicht zuletzt die aktuelle COVID-19Pandemie, wie wichtig kontinuierliches (Fern-)Monitoring und smarte DeviceApplikationen geworden sind. Prinzipiell kann man bei den Implantables und Wearables eine periodische, eine symptombezogene und eine kontinuierliche Datenerfassung vornehmen (• Tab. 2). Die periodische Datensammlung, etwa bei der Blutdruckmessung, kann durch Wearables insofern unterstützt werden, dass häufiger gemessen werden kann und damit das klinische Bild feiner und vollständiger wird. Die Erhebung von symptombezogenen Daten hat einen sehr hohen Stellenwert bei der Detektion und Analyse von Herzrhythmusstörungen. Aktuelle Daten zeigen, dass prinzipiell alle Devices dies in guter diagnostischer Qualität gewährleisten [21]. Eine kontinuierliche Datensammlung ohne unmittelbaren Symptombezug ist z.B. bei Patienten mit Vorhofflimmern zur Beurteilung des AF Burden sehr hilfreich und hat mutmaßlich sogar einen 


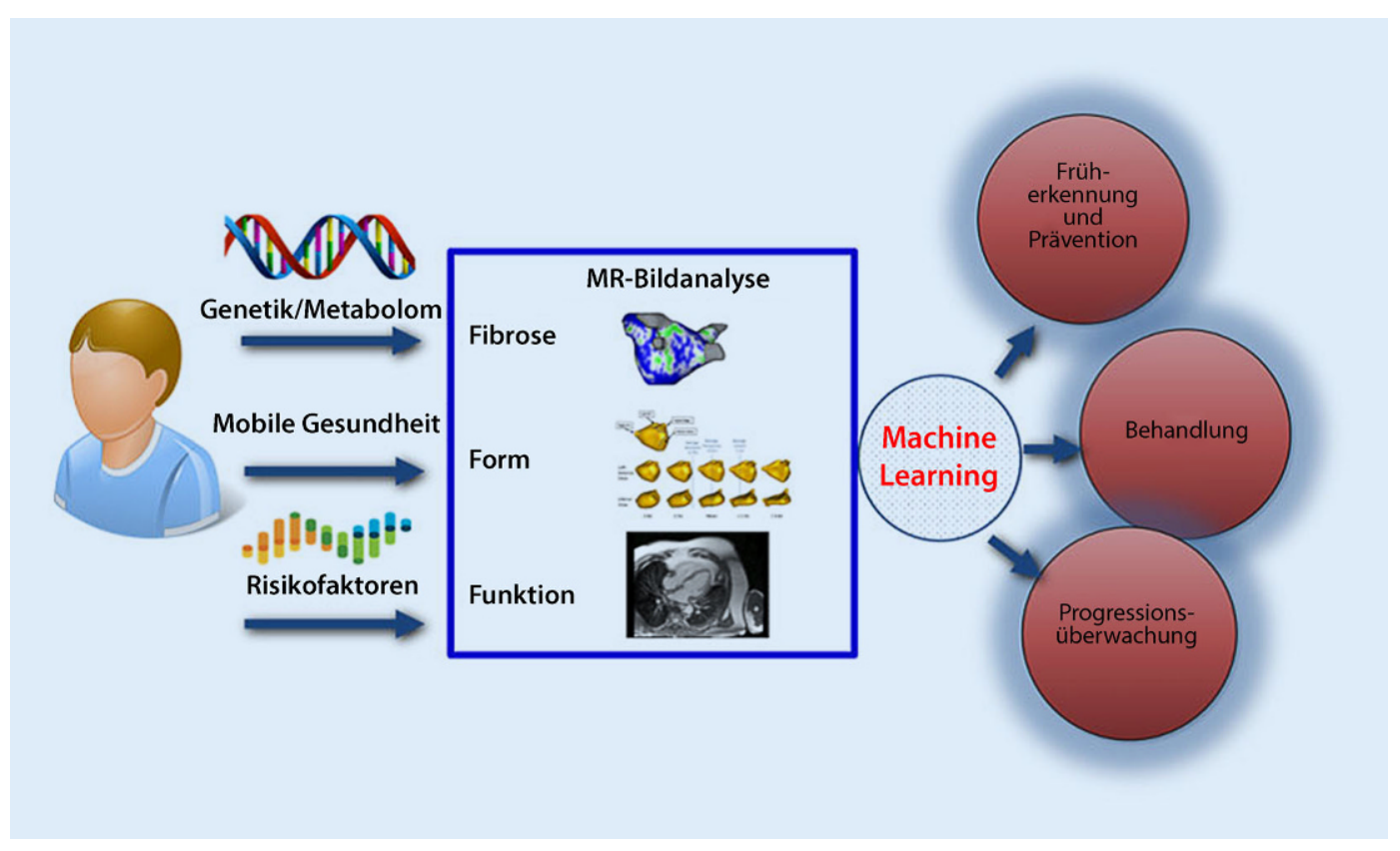

Abb. $4<$ Personalisierte Pfade bei der Therapie von Vorhofflimmern. Neben der Genetik und individuellen Risikofaktoren spielt auch die Detektion von Vorhofflimmern über smartes Monitoring (z. B. durch Wearables und Implantables) eine wichtige Rolle für individuelle Therapiekonzepte. (Nach [22], mit freundlicher Genehmigung von Oxford Academic)

prognostischen Vorteil für unsere Patienten. Asymptomatische Episoden von Vorhofflimmern haben neben zerebrovaskulären Folgen mutmaßlich auch einen Einfluss bei der Entwicklung von Fibrose und einer atrialen Kardiomyopathie [22, 23]. Daher sollte es ein primäres Ziel des kontinuierlichen Monitorings sein, auch nicht primär durch Symptome vermittelte Arrhythmie-Episoden zu erfassen. Dies ermöglicht dann auch einen personalisierten Therapieansatz (• Abb. 4).

Eine große Herausforderung für den verstärkten Einsatz von Monitoring-Devices ist weiterhin die ungefilterte Datenflut. Hier müssen zusammen mit den Herstellern ebenfalls smarte und simple Lösungen gefunden werden, um die Ereignisse anwenderfreundlich zu übermitteln und abzubilden, aber auch, um eine technische Integration in existierende ITSysteme bzw. in klinische Arbeitsabläufe einfach zu integrieren (•Tab.2). Ferner sollten Patienten darauf hingewiesen werden, dass Wearables, die kein Medizinprodukt sind, ein implantiertes Device oder ärztlich verordnetes EKG-Monitoring nicht ersetzen, sondern allenfalls ergänzen. Durch den inzwischen frei zugänglichen Markt zu Wearables, insbesondere der Smartwatches und deren assoziierten Apps, kann mit einem enormen Anstieg an Daten gerechnet werden.
Die Herausforderung für klinisch und wissenschaftlich tätige Ärzte ist es, diese Datenflut zu sortieren und zu interpretieren. Gleichzeitig ermöglicht dies z. B. die Prävalenz von asymptomatischem Vorhofflimmern in primär gesunden Menschen zu ermitteln. Dies könnte einige Vorteile bieten, etwa in der Indikation zur Antikoagulation bei kurzzeitigem asymptomatischem Vorhofflimmern.

Bei klinischen Symptomen oder besonderen Meldungen durch Wearables oder Implantables sollte weiterhin der behandelnde Arzt konsultiert werden, um die Situation zu überprüfen und adäquat reagieren zu können. Abschließend lässt sich zusammenfassen, dass Implantables und Wearables smart und simple sind und an vielen Stellen vorteilhaft in unserem klinischen Alltag eingesetzt werden können (- Tab. 3). Eine Vorstellung beim Arzt können sie aber (noch) nicht ersetzen.

\section{Fazit für die Praxis}

- Implantables und Wearables bieten eine kontinuierliche Rhythmusüberwachung unabhängig von der Mitarbeit des Patienten.

- Smartes Rhythmusmonitoring ermöglicht eine Identifikation von selten auftretenden, aber für die Pro- gnose des Patienten möglicherweise relevanten Arrhythmien.

- Die meisten aktuellen Devices zeigen eine qualitativ hochwertige und über die Zeit stabile Signalqualität.

- Fast alles Implantables und Wearables im klinischen Anwendungsgebiet bieten die Möglichkeit der telemedizinischen Anbindung.

- Die telemedizinische Anbindung der Patienten ermöglicht wiederum eine kurze Reaktionszeit, wobei datenschutzrechtliche Rahmenbedingungen und infrastrukturelle Fragen noch nicht abschließend geklärt sind.

\section{Korrespondenzadresse

PD Dr. med. Christian Sohns
Klinik für Elektrophysiologie/
Rhythmologie, Herz- und
Diabeteszentrum Nordrhein-
Westfalen, Ruhr Universität
Bochum
Georgstr. 11, 32545 Bad
Oeynhausen, Deutschland
csohns@hdz-nrw.de

\section{Einhaltung ethischer Richtlinien}

Interessenkonflikt. C. Sohns, M. Khalaph, L. Bergau und P. Sommer geben an, dass kein Interessenkonflikt besteht. 
Für diesen Beitrag wurden von den Autoren keine Studien an Menschen oder Tieren durchgeführt Für die aufgeführten Studien gelten die jeweils dort angegebenen ethischen Richtlinien.

\section{Literatur}

1. Gladstone DJ, Spring M, Dorian P, Panzov V, Thorpe KE, Hall J, Vaid H, O'Donnell M, Laupacis A, CoteR, Sharma M, Blakely JA, Shuaib A, Hachinski V, Coutts SB, Sahlas DJ, Teal P, YipS, Spence JD, Buck B, Verreault $S$, Casaubon LK, Penn A, Selchen D, Jin A, Howse D, Mehdiratta M, Boyle K, Aviv R, Kapral MK, Mamdani M, EMBRACE Investigators and Coordinators (2014) Atrial fibrillation in patients with cryptogenic stroke. N Engl J Med 370(26):2467-2477. https://doi.org/10.1056/ NEJMoa1311376

2. Sanna T, Diener HC, Passman RS, Di Lazzaro V, Bernstein RA, Morillo CA, Rymer MM, Thijs V, Rogers T, Beckers F, Lindborg K, Brachmann J, CRYSTAL AF Investigators (2014) Cryptogenic stroke and underlying atrial fibrillation. N Engl J Med370(26):2478-2486. https://doi.org/10.1056/ NEJMoa 1313600

3. Marrouche NF, Rhew D, Akoum N, Dohse $H$, Faranesh T, Leahy RA, Li J, Peters NS, Quashie R, Tarakji KG, Turakhia MP, WursterCJ(2020) Guidance for Wearable Health Solutions Kongressbeitrag: CES, Las Vegas 09012020

4. Mittal S, Pokushalov E, Romanov A, Ferrara M Arshad A, Musat D, Preminger M, Sichrovsky T, Steinberg JS (2013) Long-term ECG monitoring using an implantable loop recorder for the detection of atrial fibrillation after cavotricuspid isthmus ablation in patients with atrial flutter. Heart Rhythm 10(11):1598-1604. https://doi.org/ 10.1016/j.hrthm.2013.07.044

5. Bergau L, Sohns C, Sossalla S, Munoz-Exposito $P$, Luethje L, Zabel M (2015) Submuscular implantation of insertable cardiac monitors improves the reliability of detection of atrial fibrillation. J Interv Card Electrophysiol 42(2):143-149. https://doi. org/10.1007/s10840-014-9970-3

6. Tada S, Shibata M, Ohno S, Haruki Y, Murakami H, HottaD,NojimaM, RuhnkeGW(2018) Investigation on the optimal implantation site and setting of Reveal LINQ((R)) avoiding interference with performance of transthoracic echocardiography. J Arrhythm 34(3):261-266. https://doi.org/10. 1002/joa3.12037

7. Piccini JP, Mittal S, Snell J, Prillinger JB, Dalal N, Varma N (2016) Impact of remote monitoring on clinical events and associated health care utilization: a nationwide assessment. Heart Rhythm 13(12):2279-2286. https://doi.org/10. 1016/j.hrthm.2016.08.024

8. Klersy C, Boriani G, De Silvestri A, Mairesse GH, Braunschweig F, Scotti V, Balduini A, Cowie MR Leyva F, Health Economics Committee of the European Heart Rhythm Association (2016) Effect of telemonitoring of cardiacimplantable electronic devices on healthcare utilization: a meta-analysis of randomized controlled trials in patients with heartfailure. Eur JHeartFail 18(2):195-204.https:// doi.org/10.1002/ejhf.470

9. Pujdak K (2016) Implantable loop recorder of the Confirm family (St. Jude Medical). Herzschrittmacherther Elektrophysiologie 27(4):341-344. https://doi.org/10.1007/s00399-016-0468-9

10. Nölker G, Mayer J, Boldt LH, Seidl K, Van Driel V, Massa T, Kollum M, Brachmann J, Deneke T,
Hindricks G, Jung W, Brunner KJ, Kraus S, Hummer A, Lewalter T (2016) Performance of an implantable cardiac monitor to detect atrial fibrillation: results of the DETECT AF study. J Cardiovasc Electrophysiol 27(12):1403-1410. https://doi.org/10.1111/jce.13089

11. CiconteG, Saviano M, Giannelli L, Calovic Z, Baldi M, Ciaccio C, Cuko A, Vitale R, Giacopelli D, Conti M, Lipartiti F, Giordano F, Maresca F, Moscatiello M, Vicedomini G, Santinelli V, Pappone C (2017) Atria fibrillation detection using a novel three-vector cardiac implantable monitor: the atrial fibrillation detect study. Europace 19(7):1101-1108. https:// doi.org/10.1093/europace/euw181

12. Lewalter T, Jilek C (2016) Implantable loop recorder BioMonitor 2 (Biotronik). Herzschrittmacherther Elektrophysiologie 27(4):337-340. https://doi. org/10.1007/s00399-016-0469-8

13. Ooi SY, Ng B, Singarayar S, Hellestrand K, Illes $P_{\text {, }}$ Mohamed U, Razak S, Weerasooriya R, Biomonito 2 Study Investigators (2018) BioMonitor 2 pilot study: early experience with implantation of the Biotronik BioMonitor 2 implantable cardiac monitor. Heart Lung Circ 27(12):1462-1466. https://doi.org/10.1016/j.hlc.2017.09.005

14. Biotronik (2019) Biotronik receives FDA clearance on BioMonitor III. https://www. cardiacrhythmnewscom/biotronik-receives fda-clearance-on-biomonitor-iii/. Zugegriffen: 24.06.2020

15. Steinhubl SR, Waalen J, Edwards AM, Ariniello LM Mehta RR, Ebner GS, Carter C, Baca-Motes K, Felicione E, Sarich T, Topol EJ (2018) Effect of a home-based wearable continuous ECG monitoring patch on detection of undiagnosed atrial fibrillation: the $\mathrm{mStoPS}$ randomized clinical trial. JAMA 320(2):146-155. https://doi.org/10. 1001/jama.2018.8102

16. Fukuma N, Hasumi E, Fujiu K, Waki K, Toyooka T, Komuro I, Ohe K (2019) Feasibility of a T-shirttype wearable electrocardiography monitor for detection of covert atrial fibrillation in young healthy adults. Sci Rep 9(1):11768. https://doi.org/ 10.1038/s41598-019-48267-1

17. Aljuaid M, Marashly Q, AIDanaf J, Tawhari I, Barakat M, Barakat R, Zobell B, Cho W, Chelu MG, Marrouche NF (2020) Smartphone ECG monitoring system helps lower emergency room and clinic visits in post-atrial fibrillation ablation patients. Clin Med Insights Cardiol 14:1179546820901508 https://doi.org/10.1177/1179546820901508

18. Perez MV, Mahaffey KW, Hedlin H, Rumsfeld JS, Garcia A, Ferris T, Balasubramanian V, Russo AM, Rajmane A, Cheung L, Hung G, Lee J, Kowey P, Talati N, Nag D, Gummidipundi SE, Beatty A, Hills MT, Desai S, Granger CB, Desai M, Turakhia MP, Apple Heart Study Investigators (2019) Largescale assessment of a Smartwatch to identify atrial fibrillation. N Engl J Med 381(20):1909-1917. https://doi.org/10.1056/NEJMoa1901183

19. Wegner FK, Kochhauser S, Ellermann C, Lange PS, Frommeyer G, Leitz P, Eckardt L, Dechering DG (2020) Prospective blinded evaluation of the smartphone-based aliveCor Kardia ECG monitor for atrial fibrillation detection: the PEAK-AF study. Eur J Intern Med 73:72-75. https://doi.org/10. 1016/j.ejim.2019.11.018

20. Treskes RW, Gielen W, Wermer MJ, Grauss RW, van Alem AP, Dehnavi RA, Kirchhof CJ, van der Velde ET, Maan AC, Wolterbeek R, Overbeek OM, Schalij MJ, Trines SA (2017) Mobile phones in cryptogenic strOke patients Bringing sIngle Lead ECGs for Atrial Fibrillation detection (MOBILE-AF): study protocol for a randomised controlled trial. Trials 18(1):402. https://doi.org/10.1186/s13063-017-2131-0

21. De Coster M, Demolder A, De Meyer V, Vandenbulcke F, Van Heuverswyn F, De Pooter J (2020) Diagnostic accuracy of R-wave detection by insertable cardiac monitors. Pacing Clin Electrophysiol. https://doi.org/10.1111/pace.13912

22. Sohns C, Marrouche NF (2020) Atrial fibrillation and cardiac fibrosis. Eur Heart J 41(10):1123-1131. https://doi.org/10.1093/eurheartj/ehz786

23. Bisbal F, Baranchuk A, Braunwald E, Bayes de Luna A, Bayes-Genis A (2020) Atrial failure as a clinical entity: JACC review topic of the week. J Am Coll Cardiol 75(2):222-232. https://doi.org/ 10.1016/j.jacc.2019.11.013

24. Raja JM, Elsakr C, Roman S, Cave B, Pour-Ghaz I, Nanda A, Maturana M, Khouzam RN (2019) Apple watch, wearables, and heart rhythm: where do we stand? Ann Transl Med 7(17):417. https://doi.org/ 10.21037/atm.2019.06.79

25. Chan PH, Wong CK, Poh YC, Pun L, Leung WW, Wong YF, Wong MM, Poh MZ, Chu DW, Siu CW (2016) Diagnostic performance of a smartphonebased photoplethysmographic application for atrial fibrillation screening in a primary care setting.J Am Heart Assoc. https://doi.org/10.1161/ JAHA. 116.003428

26. Turakhia MP, Desai $M$, Hedlin $H$, Rajmane $A$, Talati N, Ferris T, Desai S, Nag D, Patel M, Kowey P, Rumsfeld JS, Russo AM, Hills MT, Granger CB, Mahaffey KW, Perez MV (2019) Rationale and design of a large-scale, app-based study to identify cardiacarrhythmiasusing a smartwatch:The Apple Heart Study. Am Heart J 207:66-75. https://doi. org/10.1016/j.ahj.2018.09.002

27. Bumgarner JM, Lambert CT, Hussein AA, Cantillon DJ, Baranowski B, Wolski K, Lindsay BD, WazniOM, Tarakji KG (2018) Smartwatch algorithm for automated detection of atrial fibrillation. J Am Coll Cardiol 71(21):2381-2388. https://doi.org/10. 1016/j.jacc.2018.03.003

28. Desteghe L, Heidbuchel H (2017) 'Performance of handheld electrocardiogram devices to detect atrial fibrillation in a cardiology and geriatric ward setting: authors' response. Europace 19(8):1408-1409. https://doi.org/10.1093/ europace/euw237

29. Koshy AN, Sajeev JK, NegishiK, Wong MC, Pham CB, Cooray SP, Khavar Y, Roberts L, Cooke JC, Teh AW (2018) Accuracy of blinded clinician interpretation of single-lead smartphone electrocardiograms and a proposed clinical workflow. Am Heart J 205:149-153. https://doi.org/10.1016/j.ahj.2018. 08.001 